\title{
Redefining the primary
} nurse role in oncology care: A 21st century perspective

\author{
By Sandra McFarlane and Cathy Bennett
}

\section{Acknowledgements}

The authors would like to recognize members of the Primary Nurse Role Development Project: Sue Bruch, RN, CON(c), Martha Finn, $B S c N, M E d, A C N P, C O N(c)$, Betty Ann Griffiths, BScN, $M S c N$, CON(c), Brenda Kowaleski, BScN, CCRC, Kristine Leach, BScN, CON(c), Nancy Ross, BHScN, CON(c), Carol Robertson, BScN, Kathy Smith, RN, CON(c) and Charlene Schell, BScN, CON(c). Sandy McFarlane, BScN, MHSc, was the project executive and Cathy Bennett, BScN, MEd, was the project leader. Juravinski Cancer Centre, Hamilton Health Sciences, Hamilton, Ontario.

\section{Abstract}

Oncology nursing is a rapidly evolving specialty with increasing demands upon nurses to respond to the complex needs of cancer patients and their families. Primary nursing $(P N)$ has been the model of care delivery utilized at our cancer centre for more than two decades. The nursing department determined it was time that a review and redefining of the role be undertaken. These objectives were achieved through the implementation of the Primary Nurse Role Development Project. This article discusses: a brief background of why the existing role needed to be reviewed and revised; an overview of how primary nursing has been applied historically at the centre; and details regarding the project plan and its implementation. Results from the project provided a competency-based oncology primary nurse role description, which will be outlined along with proposals for improving nursing practice. Our goal is to achieve optimal care for our patients.

Oncology nursing is a rapidly evolving specialty creating increased demands upon nurses to respond to the escalating complex needs of cancer patients and their families. The model of care delivery with the potential to fulfill this challenge is the primary nursing model (PN). This model is designed to facilitate nurses to practise to their fullest scope in meeting patient needs, including physical and supportive care requirements. Since primary nursing, in collaboration with oncologists, has been in practice at our large ambulatory cancer centre for more than two decades, we determined it was timely to review and redefine the role. This objective was accomplished through the implementation of the Primary Nurse Role Development Project (the project). It was positioned as a key strategic priority for the nursing department in our quest to improve not only patient care outcomes, but also to enhance the professional practice of oncology nurses at our centre. The results of the project indicated that while primary nurses functioned with relative effectiveness as demonstrated in a patient satisfaction survey (2003), enhancements to practice are required to provide optimal nursing care to individuals affected by cancer in the 21 st century.

Sandra McFarlane, BScN, MHSc, Director of Medicine Health Services, Toronto East General Hospital. Formerly Director of Nursing \& Patient Services, and Chief of Nurse Practice, Juravinski Cancer Centre at Hamilton Health Sciences.

Cathy Bennett, BScN, MEd, Education Coordinator, Juravinski Cancer Centre at Hamilton Health Sciences, Cathy.Bennett@hrcc.on.ca
This article will discuss a brief background of why the existing role needed to be reviewed and revised, an overview of how primary nursing has been applied historically at the centre, and details regarding the project plan and its implementation. Results from the project provided a competency-based oncology primary nurse role description, which will be outlined along with proposals for improving nursing practice.

\section{Why the change was needed?}

Primary nursing has been utilized at our cancer centre for many years as the model of care delivery system, but a number of factors prompted the review process, which was initiated in 2003. First, there had never been a formal evaluation of our model of care, so it was difficult to determine in absolute terms the model's effectiveness in meeting patient care needs, particularly given the fast pace of practice changes in recent years. Second, a number of physicians at the centre expressed concerns with the level of practice and expertise of some of the primary nurses who had been assigned to work with them. They indicated uncertainty as to what level of practice to expect from nurse to nurse notwithstanding the centre's criteria of only hiring nurses with a minimum of three years oncology experience and providing at least one month of orientation to all new nurses. Third, it became evident there was much variation in practice amongst the primary nurses in terms of how their overall role was implemented. The lack of standardization regarding $\mathrm{PN}$ role responsibilities had contributed to a degree of confusion, particularly when nurses were asked to cover for each other's patient groups. The last factor that prompted the review process was from a historical perspective, where more emphasis had been placed on primary nursing as a pattern of arranging nursing assignments. The focus had tended to be on functional duties within the traditional medical model approach to care. In our organizational structure, barriers existed in the delivery of care, particularly between the outpatient and inpatient areas. It became apparent that an enhanced PN model would be an important strategy to strengthen the integration of care across the hospital, ambulatory cancer centre and in the community. From a nursing perspective, there was a greater need to work toward a broader view of community to include the community hospital programs, home care and supportive care networks. The 21 st century PN role at our centre needed to embrace an enhanced focus on a patient-centred approach with more attention given to comprehensiveness and coordination of patient care.

Many of the challenges just described existed because there was no clearly documented PN role description encompassing the primary nurse core competencies. Also, there was the absence of a nursing specific performance development tool.

This project was initiated following the publication of the Canadian Association of Nurses in Oncology (CANO) Standards of Care document (2002) and it was apparent that any oncology nurse role review would have to be done in concert with the various nursing roles outlined in the document, thus ensuring alignment with the standards. With these background factors in mind, a project plan was developed.

\section{Project plan}

The project team was formed with representation from key stakeholders in the nursing department including primary nurses and other nurses from various clinical areas, a nurse manager, advanced 
practice nurses, the education coordinator leading the project and the director of nursing as the project executive. The team initiated a review and redefining of the role in order to strengthen existing nursing practices through three main objectives: 1. To clearly establish and articulate the core competencies and responsibilities of the primary nurse role, 2 . To establish a performance development process and tool based on the new role description, and 3 . To disseminate the expanded scope of the primary nurse role throughout the centre's staff including physicians and allied health.

Detailed strategies on how to achieve the expected outcomes for each objective were identified. Various milestones and the timelines for meeting the targets were established as well as assigning accountabilities. Strategies in redefining the role included reviewing of relevant literature, observation of primary nurses in action, and consultation with other organizations where primary nursing is practised. A communication plan was completed to include regular updates for all nursing staff though meetings, presentations, e-mails and written material for the duration of the project. Verbal and written feedback from the staff was integrated. The project commenced during the first quarter of 2003. Bi-weekly meetings were conducted. The project was completed within a 12-month period.

\section{The creation of the primary nurse role description}

The new primary nurse role description was created based upon the following three key principles: evidence-based, standard-based and competency-based. Available evidence was reviewed to ensure the comprehensiveness of the role description. The description incorporates relevant nursing standards as well as two specific RNAO practice guidelines (2002) and is written in a competency-based format ensuring that each competency is observable and measurable.

The most relevant information, pertinent to defining a new role description, was gathered and synthesized by project members from three key sources including: the literature regarding primary nursing, the CANO Standards document, 2002, and an articulated list of valued elements of primary nursing as expressed by our nursing staff. In the next section of this article, the three key sources will be described in more depth.

\section{Working definitions of primary nursing}

One of the initial steps in redefining the oncology PN role involved examining how it was defined and applied in the literature. The PN model was originated more than 30 years ago by a group of nurses from the University of Minnesota led by Marie Manthey. They were responding to their dissatisfaction with fragmented care and lack of consistent patient contact. According to Manthey (2002, 1988, 1980), the outcomes of team and functional nursing models precipitated the development of the PN model. Manthey states that the concept of primary nursing comprised four elements of work organization: responsibility for managing nursing is clearly allocated to an individual nurse; assignment of whole patients, rather than tasks; direct communication among caregivers; and management through leadership, rather than control. PN is a care delivery model that is viewed as professional and patient-centred (Bond, 1991). While the primary nursing delivery system emphasizes the care of the patient being assigned to one nurse, the philosophy of PN emphasizes a therapeutic nurse-to-patient relationship, and the importance of patient-centred care (Reed, 1988; Manthey, 1988; Miller, 1998). The fact that $\mathrm{PN}$ is considered to be both a philosophy of care as well as a model of care delivery is congruent with our nurses' belief system and their desired practice standards. We consulted extensively with Doris Grinspun, the Executive Director of the Registered Nurses Association of Ontario, who is considered a leading expert in primary nursing. Grinspun has explored the profound impact that primary nursing has on the patient and on the nurse-patient relationship (Stade, 1995). In a formal presentation at our centre in 2004, Grinspun emphasized that for PN to be in effect, there are two fundamental conditions, structural and substantive, which must be in place. The structural goal of PN is that every patient has a primary nurse and every nurse has primary patients. The substantive goal is to provide in-depth care that is relevant to patient and family needs. This is further reinforced by the development panel of the Client Centred Care - Nursing Best Practice Guideline (RNAO, 2002), which strongly recommends PN as the preferred model of care delivery. The overall goal of PN is the delivery of comprehensive, continuous, coordinated and individualized patient care (Grinspun, 2004; Ryan \& Logue, 1998). Many articles describe the primary nursing delivery model in hospital wards (Webb \& Pontin, 1997; Ryan \& Logue, 1998; Villaire, 1993; Sandhu et al., 1992; Manthey, 2002). Only four articles were found to address this system in an ambulatory oncology setting (Walter \& Robinson, 1994; Johnson, 1987; Paradise \& Kendall, 1985; Farley, 1981). These articles were beneficial to review by providing a historical perspective to the evolution of the primary nursing model of care in an ambulatory oncology setting, but provided minimal assistance in developing our new PN role description necessary for the 21 st century.

The second major source of content came from the document "Standards of Care, Roles in Oncology Nursing and Role Competencies" CANO (2002), which outlines the scope of practice and responsibilities of Canadian nurses interacting with patients living with cancer. The standards of care and related nursing roles and responsibilities outlined in this document provided an invaluable foundation to the project team in developing the role description. The nine standards that are threaded throughout the entire PN role description are: individualized and holistic care; family-centred care; self-determination and decision-making; navigating the system; coordinated and continuous care; supportive and therapeutic relationships; evidence-based care; professional care; and leadership. Standards from the College of Nurses of Ontario (2003, 2002, 2000, $1999 \&$ 1996) and the American Nurses Association and Oncology Nursing Society (1996) were also instrumental in building the role description, especially with regard to the competency development section.

The third and last major source of content was the articulated list of 14 valued elements of PN as expressed by members of the primary nurse project team through a purposeful project team meeting. These were: continuity of care, coordination of care, authority, advocacy, autonomy, collaborative practice, patient-centred care, decentralized decision-making, family-centred care, holistic care, leadership, supportive care, direct care and patient education.

Collating and synthesizing content from the three sources produced a total of 23 elements to describe the essence of primary nursing. The project team then defined each element carefully through a consensus process. This was achieved by distributing the 23 elements amongst the members of the project team for them to define, using current nursing resources such as Standards of Care, Roles in Oncology Nursing and Role Competencies CANO, 2002, RNAO Nursing Best Practice Guidelines (2002), and College of Nurses of Ontario Standards $(2003,2002,2000,1999)$. At subsequent project team meetings, each element definition was then presented to the team members and either accepted or revised. This process was followed to ensure a clear understanding and acceptance of the elements by the whole team. Definitions and revisions were noted in project team meeting minutes. The list of defined elements was collated into a document entitled "The Primary Nursing Elements Document", which was circulated to all centre nursing staff for their feedback. Minor revisions were incorporated based on the comments of the staff. This formed the foundation for developing the primary nursing role description. Following this work, it was evident to us that common threads from the elements needed to be assembled into a manageable format. We further organized the elements by grouping them into six major themes as shown in Figure One. 
After additional refinement, the elements were further grouped and classified into the six core domains of practice that we determined were essential to the primary nurse role as shown in Figure Two.

Once the domains were clearly defined, the project team proceeded to develop the detailed competencies associated with each of the six domains to form the primary nurse role description as seen in Figure Three (see page 102).

\section{Implications for practice and future}

The new primary nurse role description is designed to elevate standards of practice in the provision of enhanced oncology nursing care to individuals and their families affected by cancer in the $21 \mathrm{st}$ century. By clearly stating PN role expectations, variations in practice should be minimized. The new role accountabilities are targeted for promoting fuller autonomy within the scope of nursing practice to facilitate professional growth of nursing staff. To optimize patient care outcomes, the utilization of all six domains of practice of the role description is essential. Benefits in addition to improved patient care will include enhanced quality of work life, greater job satisfaction and retention of nurses.

\section{Figure One. Primary Nursing: Elements}

1. Clinical Practice Direct Comprehensive Care

Evidenced-based practice

Navigating the System

Collaborative Practice

2. Philosophy of Care

Patient-Centred Care

Family-Centred Care

Coordinated Care

Continuity of Care

3. Professional Practice

Decentralized Decision-Making

Ethics in Nursing

4. Leadership

Autonomy

Accountability

Authority

5. Supportive Care

Patient Education

Advocacy

6. Evaluation

Reflective Practice

Evaluate outcomes

\section{References}

American Nurses Association and Oncology Nurses Society. (1996). Statement on the scope and standards of oncology nursing practice.

Bond, S. (1991). Evaluating primary nursing. Nursing Standard, 5(36), 35-39.

Canadian Association of Nurses in Oncology. (2002). Standards of care, roles in oncology nursing and role competencies. Canadian Oncology Nursing Education Project.

College of Nurses of Ontario. (2003, Dec.). Building your leadership skills. Communique. Toronto, ON.
A communication plan was implemented including presentations, meetings and e-mails to ensure the entire nursing staff understood the new role description. Dissemination of information with physicians and other disciplines in the centre was established to ensure all stakeholders were informed of the expanded PN role. A Grand Rounds, attended by many disciplines including the hospital and community, was provided to explain the project work. Also, a presentation was made at the annual Canadian Association of Oncology Nurses Conference in Calgary in October 2004.

To assist nurses in the implementation of the new role, educational initiatives are being formulated to ensure all current and new staff develop the competencies within each of the six domains of practice over the next few years. Since the learning needs of each nurse will differ, a PN self-assessment tool based on the competencies of the new role description and modeled on the College of Nurses of Ontario Self-Assessment Tool (1996) has been developed. Once the nurses' learning needs are identified, through each nurse completing the primary nurse self-assessment tool, the data will be analyzed to facilitate the development of customized educational activities to advance the oncology primary nursing practice.

The new PN role description created the requirement for a performance development process and tool to monitor the nurses' progress in the application. A performance development tool was developed integrating the new PN role description. This was the second key result of the project. The nursing profession needs performance development tools to promote ongoing professional standards, staff development and ensure competence in the provision of patient care.

This project has been extremely valuable and worthwhile to the professional growth of our nursing staff.

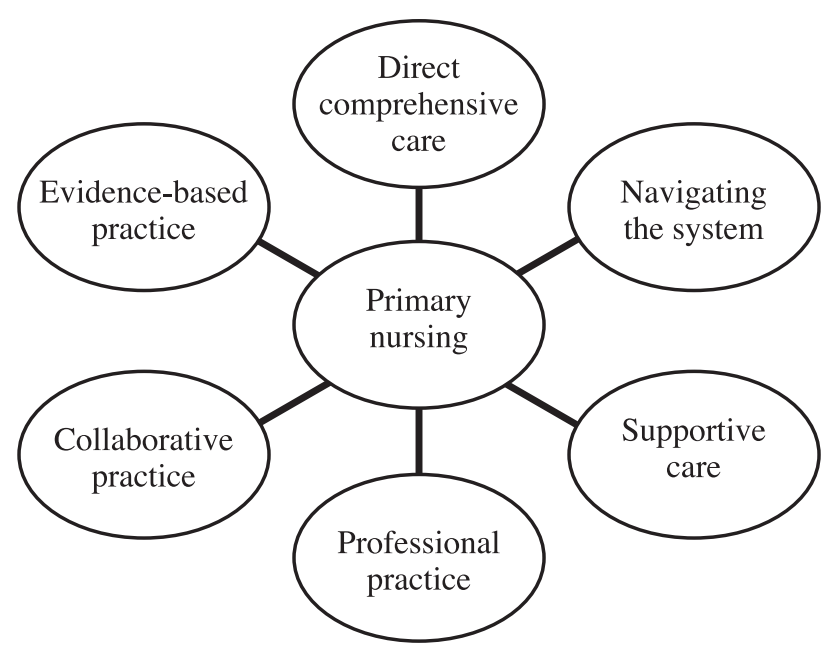

Figure Two. 
Farley, B. (1981). Primary nursing in the oncology ambulatory setting. Nursing Administration Quarterly, Summer, 44-53.

Grinspun, D. (2004). Primary nursing: What patients and nurses need and deserve. Disease Site Team Rounds, May 4. Juravinski Cancer Centre, HHS, Hamilton, ON.

Johnson, C. (1987). Primary nursing model fosters staff's autonomy and accountability. Dimensions, 64, 30-31.

Manthey, M. (2002). The practice of primary nursing (2nd ed.). Minneapolis, MN: Creative Healthcare Management.

Manthey, M. (1980). The practice of primary nursing. U.S.: Blackwell Scientific Publications, Inc.

Manthey, M. (1988). Can primary nursing survive? American Journal of Nursing, 88, 644-647.

Miller, D. (1998). Thirty years of primary nursing. Creative Nursing, 2, 3-4.

Paradise, R., \& Kendall, V. (1985). Ambulatory care: Primary nursing brings continuity. Nursing Management 16, 27-30.

Reed, S. (1988). A comparison of nurse-related behaviour, philosophy of care and job satisfaction in team and primary nursing. Journal of Advanced Nursing. 13(3), 383-395.

RNAO. (2002). Establishing therapeutic relationships. Nursing Best Practice Guideline. Toronto, ON.
RNAO. (2002). Client-centred care. Nursing Best Practice Guideline. Toronto, ON.

Ryan, A., \& Logue, H. (1998). Developing an audit tool for primary nursing. Journal of Clinical Nursing, 7(5), 417-423.

Sandhu, B., Kerouac, S., Duquette, A., \& Turchon, S. (1992). Nursing assignment patterns and patient outcomes. Can J of Nurs Adm, 5, 14-19.

Stade, B. (1995). The First Provincial Primary Nursing Conference. Registered Nurse Journal, Aug./Sept, 17.

Supportive Care Research Unit, Juravinski Cancer Centre of Hamilton Health Sciences. (2003). Patient satisfaction survey, 2003. Hamilton, ON.

Villaire, M. (1993). Marie Manthey on the evolution of primary nursing. Critical Care Nurse, 13, 100-107.

Walter, J., \& Robinson, S. (1994). Nursing care delivery models in ambulatory oncology. Seminars in Oncology Nursing, 10(4), 237-244.

Webb, C., \& Pontin, D. (1997). Evaluating the introduction of primary nursing: The use of a care plan audit. Journal of Clinical Nursing, 6(5), 395-401.

\section{Figure Three. Job Description: Primary Nurse \\ PURPOSE}

The nurse practises within a primary nursing model, performing at a high level of autonomy and accountability in collaboration with oncologists and allied health professionals. He/she provides patient care from prevention, early detection, diagnosis, treatment, follow-up, palliation and bereavement. Reporting to a clinical nurse manager, the primary nurse provides patient-centred care for oncology patients and their families through direct comprehensive and supportive care, navigating the system, maintaining professional practice, and through collaborative and evidence-based nursing practice.

\section{DUTIES \& RESPONSIBILITIES}

This role demonstrates oncology-nursing expertise in managing disease site focused populations of patients with cancer.

The principal responsibilities include:

- Direct Comprehensive Care

- Supportive Care

- Navigating The System

- Professional Practice

- Collaborative Practice

- Evidence-Based Practice

\section{Direct Comprehensive Care}

Applies an integration of theory, research, experience and practical knowledge in providing holistic care for patients with cancer and their families to a maximum scope of practice through the use of protocols, practice guidelines/care paths and medical directives.

- Conducts a thorough health assessment of the patient's physical, psychosocial, cultural and spiritual needs by completing a patient history and physical examination.

- Formulates and implements a holistic plan of nursing care that is communicated and clarified with the patient/family.

- Interprets and recommends diagnostic tests and procedures when nursing judgment and expertise is required.

- Demonstrates competent clinical skills in a variety of settings.

- Effectively manages care over the telephone with patient/family and community health care professionals, enabling them to navigate the health care system.

- Conducts patient education consisting of: assessment of learning need, development of learning objectives with patient, implementation of teaching, and evaluation of learning.

- Applies adult learning principles in the provision of education through a variety of approaches and strategies.

- Provides care that demonstrates sensitivity to client diversity and incorporates appropriate cultural practices.

- Maintains knowledge of current clinical trials relevant patient population being served and communicates effectively with clinical trials staff to promote continuity of care.

- Reviews clinical trials protocols that may impact on overall patient care.

- Evaluates patient responses to treatment in order to determine progress and expected outcomes of care. Modifies plan of care accordingly.

- Provides a comprehensive report when transferring accountability of a patient's care to another nurse and/or facility.

- Documents/dictates patient history, physical examination data, nursing care plan and interventions, and the client's responses in the client's record in a timely manner in accordance with the CNO Standards and established documentation standards.

Figure continued on page $103 .$. 


\section{Supportive Care}

Provides continuous and coordinated nursing care to meet the emotional, psychological, informational, spiritual and practical needs of patients throughout the cancer journey.

- Establishes a caring and supportive relationship with patients demonstrating trust, respect and honesty.

- Demonstrates advanced communication skills with each patient encounter.

- Creates a healing environment by active listening and offering appropriate support.

- Demonstrates the ability of "being there" for the patient throughout their illness by acknowledging the patient's reality and encouraging hope.

- Assists the patient to identify psychosocial concerns and offers appropriate resources through information, education and referral.

- Demonstrates counselling skills in assisting patient and family to deploy a variety of coping mechanisms throughout the continuum of care from diagnosis to bereavement.

\section{Navigating the System}

Navigation is used to denote a proactive nursing role intended to expedite access to services and resources, and to improve continuity and coordination of care to manage the complex maze of cancer treatment and care. The nurse empowers the patient and family by providing information and education to facilitate decision-making. This enhances patient's choice in determining the best alternatives of care to meet their individual needs.

- Knowledgeable about the resources available at the Juravinski Cancer Centre, Hamilton Health Sciences, Central West Region Community Oncology Care Services, and Community Care Access Centres.

- Advocates for the patient and family by mobilizing appropriate resources tailored to the individual patient's care in a timely fashion. Identifies gaps in service and addresses them.

- Organizes the interdisciplinary plan of care with team members as required to facilitate the seamless movement of the patient through the cancer care system.

- Understands the full impact of the illness and strategizes when and how best to guide the patient and family throughout the cancer treatments.

- Develops a positive relationship with patients and families that are respectful of their needs, wishes, knowledge, experience, values and beliefs.

- Translates medical language and procedures for patients and family by using language that is understandable for the patient.

\section{Professional Practice}

Nurses are accountable for their own decision-making and actions, and maintaining competence to provide the best possible nursing care for patients.

- Participates in professional and specialty nursing organizations.

- Supports development of and participates in clinically relevant research, committee and project work.

- Assists in the development, implementation and evaluation of quality improvement initiatives (policy \& procedure, standards, protocols, clinical pathways and medical directives).

- Engages in self-appraisal by being proactive in meeting own learning needs.

- Engages in reflective practice, acquires ongoing knowledge and skill that will advance oncology nursing practice.

- Role models as oncology nursing expert.

- Functions as a resource within the centre, hospital and community agencies to improve nursing care and practice.

- Acts as a preceptor or mentor to staff/students, providing written/verbal feedback as necessary.

- Recognizes ethical dilemmas and works through them effectively using resources and a logical approach.

- Demonstrates ethical behaviour (i.e. honesty, integrity).

- Recognizes when own values and beliefs conflict with the patient's and is able to demonstrate conflict resolution skills.

\section{Collaborative Practice}

In collaborative practice, the primary nurse and oncologist partner in the care of the patient through a complementary relationship, which is based on shared decision-making and joint problem solving. Both the nurse and oncologist utilize their skills and expertise to work in partnership with allied health professionals to provide comprehensive care to patients and families.

- Demonstrates an understanding of nursing scope of practice and delivers care to the full scope by ensuring sound knowledge of role clarity, autonomy and accountability.

- Utilizes strong communication and interpersonal skills to facilitate mutual agreement and acceptance of responsibilities utilizing a team approach.

- Facilitates cost-effective care through complementary, but non-duplicating roles.

- Enables the patient and members of the multidisciplinary team to participate in the planning, implementation and evaluation of care.

- Manages telephone-mediated care as required involving the team as necessary.

- Demonstrates conflict resolution skills by addressing practice concerns in a collegial manner.

\section{Evidence-Based Practice (EBP)}

EBP is the conscientious use of current available evidence from health care literature in making decisions about the care of patients. The primary nurse participates in evidence-based practice in order to enable the best possible outcomes for patients.

- Demonstrates understanding of theoretical and scientific knowledge that supports clinical practice.

- Applies up-to-date knowledge and critical thinking skills.

- Identifies knowledge gap and practice concerns and seeks appropriate learning.

- Demonstrates an understanding of and engages in the following five steps of evidence-based practice:

$\mathrm{n}$ Has the ability to formulate an answerable question, which is clinically relevant.

$n$ Performs a rigorous search strategy to identify the best quantitative and qualitative evidence.

$n$ Critically appraises the strength of the research evidence for its validity and relevance to clinical practice and patient care needs.

$n$ Disseminates findings to the primary team(s), integrating the evidence into practice.

Evaluates effectiveness of interventions in attaining the desired patient outcomes. 